which may be described as staggering, for now it has more than two hundred thousand monthly subscribers on its books. Mr. Piel, publisher, and his colleagues are to be congratulated on this great achievement, especially since, through sound policy, good editing and attractive presentation this journal has won the high regard of scientists and laymen alike for its excellent interpretation of scientific discoveries and advancement.

All the articles which appear in The Scientific American are authoritative, being written by men of science directly concerned with the work being reported -usually leading authorities themselves. In the past, these have included Einstein, Karl Compton, Robert Oppenheimer and Hans Bethe. But these articles are rigorously edited with the view of clarification, not simplification. The journal is lavish with its illustrations-photographs, drawings, charts and diagrams, many of which are coloured. But the editors make sure that the text shall not become a mere vehicle for attractive pictures. The aim of the latter is to give the reader a vivid grasp of what the article is about and to develop the relationships between variables which so often defy exposition by the written word. So fine, rather than commercial, artists are employed because, as the publisher claims, "The creative artist, when he turns his interest to science, tends to come up with really original solutions to the problems presented by the text".

In connexion with The Scientific American, ten paper-backed topical 'anthologies' have also been published, and the aim is to issue about five of these every eighteen months. These 'anthologies' have already been translated into a dozen languages.

Here is a very successful venture into scientific literature for which all scientific workers and especially non-scientific readers must be very grateful. To mark the tenth year of publication under its new direction, a "Cumulative Index" (author, title and subject) of all the issues of this magazine from its first issue (May 1948) to 1957 is now available. We take this opportunity of congratulating the board of editors, including Mr. Gerard Piel (publisher), Mr. Dennis Flanagan (editor) and Mr. Leon Svirsky; the art director, Mr. James Grunbaum; the general manager, Mr. Donald Miller, jun.; and the advertising manager, Mr. Martin M. Davidson, on their magnificent achievement. They are making a first-class and essential contribution to the advancement of science.

\section{Medical Colour Television}

ON.June 4 the first mobile medical colour television unit to be designed and manufactured in Great Britain was handed over by the Marconi's Wireless Telegraph Co., Ltd., to Smith, Kline and French Laboratories, pharmaceutical manufacturers, who ordered the equipment. The vehicle weighs 6 tons, and the television apparatus accounts for a further 2 tons. The available space has been divided into three sections. The rear compartment is staffed by one maintenance engineer to service the apparatus, which combines the red, green and blue signals for transmission via land-line or microwave link. The technical director, the vision mixer, the sound mixer and two camera-control operators work in the centre compartment under the control of the producer. This room houses a colour monitor for each camera, and a third one acts as a line monitor. These are fixed to a monorail, so that they can be moved to either of the other two sections for maintenance or repair. The driver's compartment is also used as a servicing area. The cameras are direct descendants of those supplied to the British Broadcasting Corporation a few years ago. The display unit is a Marconi Colour Television Projection Unit, which can provide a picture $8 \mathrm{ft}$. $\times 6 \mathrm{ft}$., which is said to be adequate for viewing by an audience of up to 300 . This equipment will be made available, without charge, to conferences and congresses, so that large audiences can obtain a close-up view of operations or clinical demonstrations. A two-way radio link is provided to facilitate discussion between the surgeon or demon. strator and the audience.

\section{Smoking and Lung Cancer}

Following the decision of the Ministry of Health to accept the Medical Research Council's findings as establishing a causal relationship between smoking and lung cancer, local authorities were asked to promote appropriate educational campaigns. The Central Council for Health Education was recommended as a suitable agency. To help local authorities, Dr. John Burton, director of the Central Council for Health Education, has produced a summary of the relevant medical and ethical issues which should be considered by local authorities before launching its campaign. It includes the essential facts about lung cancer; criticism of the facts; other factors involved for which there is some evidence ; facts about smoking; reasons for and attitudes to smoking; giving up smoking; policy on presentation of the case against smoking; aims and methods of presenting the facts ; and the questions people ask. The summary is a clear, objective, factual account of the relationship between smoking and lung cancer and should give local authorities the kind of lead for which so many of them have been asking.

\section{Language and Speech}

INTEREST in the problems of language and speech has grown rapidly in recent years. In this, the biggest single factor is undoubtedly modern communications engineering and its impact upon the biological sciences. New electro-acoustic techniques have thrown much light on the physical basis of speech and are being increasingly applied to the study of fundamental problems in experimental psychology. The theory of information, as developed by Shannon and others, would appear to have important application to biological systems, not excluding the field of human behaviour. Furthermore, recent developments in statistical technique have given promise of a new and more genuinely scientific approach to linguistics. In view of these considerations, any serious attempt to bring together the many and diverse approaches to the study of speech deserves a warm welcome. Language and Speech is a new quarterly edited by Dr. D. B. Fry, of the Department of Phonetics, University College, London (annual subscription: $80 s . ; 11.50$ dollars. Single issues : 25s.; 4 dollars. Teddington, Middlesex : Robert Draper, Ltd., 1958). Its avowed purpose is "... to furnish a medium for publications in which the fundamental problems of speech and language form the centre of interest". It is to offer space not only for reports of experimental findings but also for discussion of theoretical issues arising from, or relevant to, experiment. The fields covered include language structure, the transmission and reception of speech, mechanical translation, synthetic speech, and abnormalities of speech and language. Of particular interest 